\title{
Correlation lengths for vortex-liquid freezing in a model of layered high-temperature superconductor
}

\author{
A. Zamora* \\ Instituto de Ciencias Nucleares, UNAM, Apdo. Postal 70-543, México D.F. 04510
}

(Dated: November 19, 2018)

\begin{abstract}
We present results from extensive numerical simulations on the Lawrence-Doniach model within the lowest Landau level approximation in the unconventional spherical geometry. We study the in-layer pancake vortex density-density correlation function (intra-layer structure factor) and the layer-to-layer order-parameter correlation function along the direction perpendicular to the layers. Our results show strong evidence for the existence of a single first-order phase transition at which both inter-layer coupling and appearance of crystalline order in the in-layer vortex correlations take place as temperature is lowered.
\end{abstract}

PACS numbers: 74.,74.25.-q,74.72.-h

The study of the phase diagram of layered superconductors in the presence of a magnetic field has attracted much attention since the discovery of the hightemperature superconducting cuprates ${ }^{1}$. In particular, the most interesting effects are found in the mixed phase ${ }^{2}$ where the external magnetic field penetrates the sample in the form of quantized flux-lines (or vortices). Within the mixed phase, originally described by Abrikosov ${ }^{3}$ as a vortex lattice, a high-temperature phase with finite resistivity has been found and called a vortex liquid. While the vortex-liquid phase is quite narrow in conventional type-II superconductors, its width is known to become large in highly anisotropic cuprates ${ }^{4}$ (e.g. $\mathrm{Bi}_{2} \mathrm{Sr}_{2} \mathrm{CaCu}_{2} \mathrm{O}_{8}$.) Assuming a specimen completely free of defects, the low-temperature phase is expected to be the Abrikosov vortex-lattice and the phase boundary separating the vortex liquid and vortex lattice appears to be a line of first-order phase transitions usually called the melting line ${ }^{5}$ (which essentially coincides with the magnetic irreversibility-line $\left.{ }^{6}\right)$. Results from experiments, analytic calculations, and numerical simulations on several models of layered superconductors have shown that, in addition or simultaneous to melting, there is a transition associated with layer decoupling ${ }^{7-13}$. In another simulation, Wilkin and Jensen ${ }^{14}$ on a layered model find a first-order transition associated with layer decoupling followed by melting but which shows no obvious thermodynamic signature. An alternative scenario was proposed by Kienappel and Moore ${ }^{15}$. From Langevin-dynamics simulations on the Lawrence-Doniach (LD) model within the lowest Landau level (LLL) approximation and in the same spherical (S) geometry that we consider here (we shall call this the S-LD-LLL model) they find hysteretic effects on several quantities which disappear on increasing the interlayer coupling-strength. They attribute this result to a line of first-order transitions ending at a critical point which separates phases of coupled and decoupled vortex-liquid (expected to freeze only at zero temperature.)

In this paper we present an alternative view based on Monte Carlo (MC) simulations in the same S-LD-LLL model. From studies of inlayer vortex-density correla- tions as well as layer-to-layer order parameter correlations we find strong evidence for vortex-liquid freezing (simultaneous to inter-layer coupling) at a finite temperature. As in Ref. ${ }^{15}$, we model this layered superconductor by a system of $M$ concentric spherical-layers of thickness $d_{0} \ll R$, where $R$ is the average radius of the sample, and interlayer spacing $s \geq d_{0}$. The reason for the choice of this geometry, instead of the usual array of plane-layers, is that this geometry guarantees full rotational and translational invariance of the vortex system (which quasi periodic boundary conditions in the planar geometry do not). This allows for the in-layer free movement of vortices without any boundary constraint, which is expected to better describe a real superconducting sample. This has been previously discussed in detail for the single-layer case by Dodgson and Moore ${ }^{16}$. An external magnetic field $\mathbf{H}(\mathbf{r})=\left(H_{0} R^{2} / r^{2}\right) \hat{\mathbf{r}}$ [with $H_{0}$ a constant field], orthogonal to all layers in every point, is generated by an infinitely long and thin solenoid ending at the center of the concentric spheres. We neglect the fluctuations in the magnetic induction inside the sample so that it has the mean value $B=\mu_{0} H(R)$ across all superconducting layers. Flux quantization requires that the magnetic induction penetrating the sample be such that $4 \pi R^{2} B=N \Phi_{0}$, where $\Phi_{0}=h / 2|e|$ is the flux quantum and $N$ the number of flux lines or vortices. Our choice of gauge that satisfies $\mathbf{B}=\boldsymbol{\nabla} \times \mathbf{A}$ is $\mathbf{A}=\left(\mu_{0} H_{0} R^{2} / r\right) \tan (\theta / 2) \hat{\boldsymbol{\phi}}$. We assume that the system without impurities accepts a description in terms of the LD Hamiltonian functional ${ }^{17}$ which, under the previous assumptions, can be written as

$$
\begin{aligned}
\mathcal{H}\left[\psi_{n}(\mathbf{r})\right] & =\sum_{n=1}^{M} d_{0} \int \mathrm{d}^{2} r\left[\alpha(T)\left|\psi_{n}\right|^{2}+\frac{\beta}{2}\left|\psi_{n}\right|^{4}+\right. \\
+ & \left.\frac{1}{2 m_{\perp}}\left|\mathbf{D}_{\perp} \psi_{n}\right|^{2}+\frac{\hbar^{2}}{2 m_{\|} s^{2}}\left|\psi_{n+1}-\psi_{n}\right|^{2}\right] .
\end{aligned}
$$

Here $\psi_{n}(\mathbf{r})$ is the two-dimensional Ginzburg-Landau (GL) order parameter in layer $n$ and $\mathbf{D}_{\perp}=-i \hbar \boldsymbol{\nabla}_{\perp}-2 e \mathbf{A}$ the gauge covariant derivative operator (acting on the surface of the sphere). $\alpha(T)$ and $\beta$ are the usual parameters from the GL theory ${ }^{18} \cdot m_{\perp}$ and $m_{\|}$are the effective 
Cooper-pair masses in the perpendicular and parallel directions relative to the radial magnetic field. The LLL approximation consists in expanding the GL order parameter in each layer as a linear combination of the degenerate eigenfunctions of $D_{\perp}^{2}=\mathbf{D}_{\perp} \cdot \mathbf{D}_{\perp}$ corresponding to the lowest eigenvalue $2|e| \hbar \mu_{0} H$. An orthonormal set of functions for the LLL on the surface of the sphere is ${ }^{19}$

$$
\varphi_{q, N}(\theta, \phi)=\mathcal{N}_{q, N} e^{i q \phi} \sin ^{q}(\theta / 2) \cos ^{N-q}(\theta / 2),
$$

where $q=0,1, \ldots, N$ labels the degeneracy of the LLL. The normalization factor is $\mathcal{N}_{r, s}=\left[(s+1) C_{s}^{r} / 4 \pi R^{2}\right]^{1 / 2}$, with $C_{s}^{r}=s ! / r !(s-r)$ ! the binomial coefficient. We expand the superconducting order parameter in layer $n$ as $\psi_{n}(\mathbf{r})=Q \sum_{q=0}^{N} v_{n, q} \varphi_{q, N}(\theta, \phi)$ with $Q=$ $\left(\Phi_{0} k_{B} T / \beta d_{0} B\right)^{1 / 4}$ and measure lengths in units of the magnetic length $l_{m}=\left(\Phi_{0} / 2 \pi B\right)^{1 / 2}$. In these units $R=(N / 2)^{1 / 2} l_{m}$.

Within the LLL approximation, the Hamiltonian functional in Eq. (1) becomes a function of the complex coefficients $\left\{v_{n, q}\right\} \equiv \mathbf{v}$ given by

$$
\begin{aligned}
\mathcal{H}(\mathbf{v}) / k_{B} T=\sum_{n=1}^{M} & {\left[\alpha_{T} \sum_{q=0}^{N}\left|v_{n, q}\right|^{2}+\frac{1}{2 N} \sum_{p=0}^{2 N}\left|U_{n, p}\right|^{2}+\right.} \\
& \left.+\eta\left|\alpha_{T}\right| \sum_{q=0}^{N}\left|v_{n+1, q}-v_{n, q}\right|^{2}\right], \quad
\end{aligned}
$$

where the effective temperature-field parameter is ${ }^{20}$

$$
\alpha_{T}=\frac{d_{0} Q^{2}}{k_{B} T}\left(\alpha(T)+\frac{|e| \hbar \mu_{0} H}{m_{\perp}}\right) .
$$

[Note that $\alpha_{T}=0$ corresponds to the mean-field $H_{c 2}(T)$ line and $\alpha_{T}=-\infty$ corresponds to $T=0$.] The parameter that determines the coupling strength between adjacent layers is then just $\eta=\hbar^{2} /\left(2 \gamma^{2} s^{2}\left|e B \hbar+m_{\perp} \alpha(T)\right|\right)$, where $\gamma=\left(m_{\|} / m_{\perp}\right)^{1 / 2}$ is the anisotropy parameter. In the second term of Eq. (3) we have used

$$
\begin{aligned}
U_{n, p}=\left(\pi^{1 / 2} N / R \mathcal{N}_{p, 2 N}\right) & \sum_{q=0}^{N} \mathcal{N}_{q, N} \mathcal{N}_{p-q, N} v_{n, q} v_{n, p-q} \\
& \times \Theta(p-q) \Theta(N-p+q),
\end{aligned}
$$

with $\Theta(x)$ the Heaviside step function, equal to zero for $x<0$ and one for $x \geq 0$. The Hamiltonian describing our model of a layered superconductor within the LLL approximation is that in Eq. (3). Note that $\mathcal{H}$ is a function of the complex variables $\left\{v_{n, q}\right\}$ and depends on the temperature, $\alpha_{T}$, and layer-coupling, $\eta$, parameters.

The equilibrium properties of this system are determined by its partition function

$$
Z(T, H)=\int \prod_{n} \mathcal{D} \psi_{n} \mathcal{D} \psi_{n}^{*} \mathcal{D} \mathbf{A} \exp \left(-\mathcal{H}\left[\psi_{n}, \mathbf{A}\right] / k_{B} T\right),
$$

whose value is basically controlled by the order parameter configurations that minimize the Hamiltonian functional
$\mathcal{H}$. In the LLL approximation, which neglects fluctuations in the magnetic induction and restricts the order parameter (in each layer) to the set of functions spanning the LLL, the partition function can be written as

$$
Z(T, H)=\int \prod_{n, q} \mathrm{~d} v_{n, q} \mathrm{~d} v_{n, q}^{*} \exp \left[-\mathcal{H}(\mathbf{v}) / k_{B} T\right] .
$$

Thermal averages of the quantities of interest $X(T, H)$,

$$
\begin{aligned}
\langle X(T, H)\rangle=\frac{1}{Z(T, H)} \int \prod_{n, q} \mathrm{~d} v_{n, q} \mathrm{~d} v_{n, q}^{*} X(\mathbf{v}) \\
\times \exp \left[-\mathcal{H}(\mathbf{v}) / k_{B} T\right],
\end{aligned}
$$

are calculated by means of MC simulations using the standard Metropolis algorithm ${ }^{21}$. We calculate thermal averages by the Importance Sampling Method ${ }^{22}$.

The physical quantities that we have focused on to examine in-layer vortex correlations are the structure factor (in each layer) defined $\operatorname{as}^{23}$

$$
S(\mathbf{k})=\frac{1}{N}\left\langle\sum_{i, j=1}^{N} \mathrm{e}^{-i \mathbf{k} \cdot\left(\mathbf{x}_{i}-\mathbf{x}_{j}\right)}\right\rangle,
$$

where $\left\{\mathbf{x}_{l}\right\}$ are the positions of the $N$ pancake vortices (on that layer) and we have chosen to parametrize the wave vector in polar coordinates as $\mathbf{k}=\left(k_{x}, k_{y}\right)=$ $(k \cos \phi, k \sin \phi)$, and the rotationally averaged structure function (also in each layer) given by

$$
\Delta(k)=\frac{1}{2 \pi} \int_{0}^{2 \pi} \mathrm{d} \phi S(\mathbf{k}) .
$$

A Lorentzian fit to the first peak of this function is in agreement with an exponential decay of vortex densitycorrelations in real space ${ }^{16}$. The length scale governing that decay, $\xi_{D}$, is proportional to the inverse-width at half peak, $\delta^{-1}$, of the Lorentzian.

Numerical measurements of these quantities have been made for runs up to $1.92 \times 10^{6} \mathrm{MC}$ steps $^{24}$ with systems as big as 18 layers containing 72 vortices per layer. We have studied the range of effective temperatures $-13 \leq \alpha_{T} \leq 2$ (while cooling) for inter-layer couplings $\eta=0.14,0.5,1,2,10,100$. One of the most notable features of a freezing transition, which is the appearance of Bragg peaks in the structure factor $S(\mathbf{k})$, is observed at an effective temperature $\alpha_{T} \simeq-4$ (for $\eta=0.14$ ) and at higher temperatures for $\eta \geq 0.5$. Fig. 1 shows this structure factor as temperature is lowered (see caption). Fig. 1(b) corresponds to the temperature $\alpha_{T}=-4$ at which the vortex system is essentially crystallized. We notice that at and below $\alpha_{T}=-5$ (Figs. 1(c) to 1(f)) the Bragg peaks appear exactly at the same positions. This is consistent with a legitimate crystalline vortexphase for these low temperatures. Another signature of the vortex-liquid freezing is seen in the rapid growth of vortex density-correlations, $\xi_{D}$, below $\alpha_{T}=-3$ (for the same coupling $\eta=0.14$ ). This is shown in Fig. 2, 


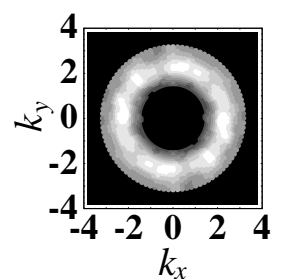

(a)

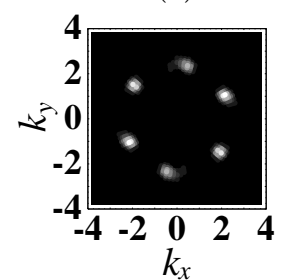

(d)

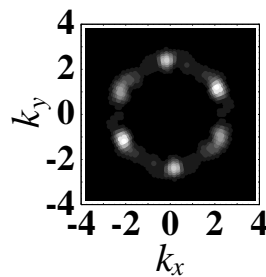

(b)

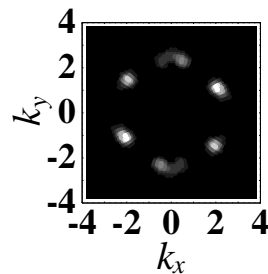

(e)

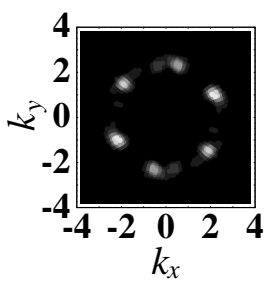

(c)

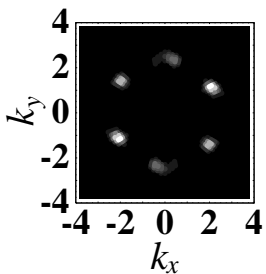

(f)
FIG. 1: Structure factor, $S(\mathbf{k})$, at layer $M / 2$ of the clean layered superconductor with interlayer coupling $\eta=0.14$ for $(N, M)=(72,18)$ as the temperature is lowered: $\alpha_{T}=$ $-3,-4,-5,-6,-7,-8 . \quad[(\mathrm{a}),(\mathrm{b}),(\mathrm{c}),(\mathrm{d}),(\mathrm{e}),(\mathrm{f})$ respectively. $]$ The central maximum at $\mathbf{k}=\mathbf{0}$ has been removed for clarity. Note the appearance of Bragg peaks at a temperature between $\alpha_{T}=-3$ and -4 .

where the scaled density correlation length $\xi_{D} / R$ is plotted. Fig. 2(a) shows the abrupt increase of this length scale as a function of our temperature parameter $\alpha_{T}$ for all system sizes studied. Fig. 2(b) shows the same characteristic length-scale, $\xi_{D} / R$, plotted (in log-linear scale) against $\left|\alpha_{T}\right|^{2}$. We observe that an exponential fit (straight-line there) to the data is appropriate at high temperatures (i.e. at low values of $\left|\alpha_{T}\right|^{2}$ ), but breaks down in the low temperature regime where correlations grow even faster than that. The exponential behavior, $\xi_{D} \sim \exp \left(\right.$ const $\left.\left|\alpha_{T}\right|^{2}\right)$, has been predicted by Moore ${ }^{25}$ in connection with bulk anisotropic-superconductors in the continuum limit at their lower critical dimension. In that investigation, however, this rapid growth of correlations is not attributed to a thermodynamic phase transition but just to a crossover. Returning to Fig. 2 (b), we remark that the very last points mark the onset of in-layer vortex correlations growing even faster than $\xi_{D} \sim \exp \left(\right.$ const $\left.\left|\alpha_{T}\right|^{2}\right)$ [compare with Fig. 2(a) for lower temperatures.] We believe this can occur because a thermodynamic first-order transition takes place at the temperature $\alpha_{T, m}$ where $\xi_{D}$ departs from the straight line in Fig. 2(b). This takes place at $\alpha_{T, m}=-3.25$ for $\eta=0.14$. In fact, comparison with the work of $\mathrm{Hu}$ and MacDonald $^{12}$ gives excellent agreement for the melting (freezing) transition at these values.

To study pancake-vortex correlations along the axis perpendicular to the layers (the $c$-axis), we measure numerically the two-point correlation function

$$
C_{P}(m)=\frac{4 \pi R^{2}}{Q^{2}}\left\langle\frac{1}{M} \sum_{n=1}^{M} \overline{\psi_{n}^{*}(\mathbf{r}) \psi_{n+m}(\mathbf{r})}\right\rangle .
$$

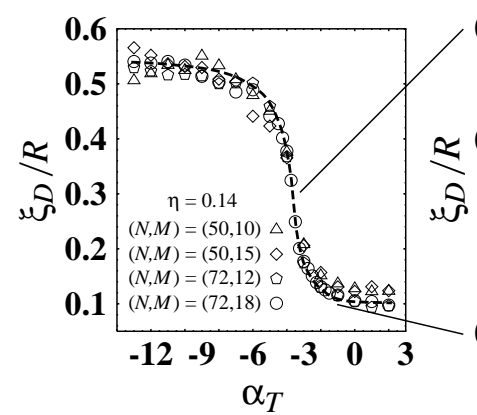

(a)

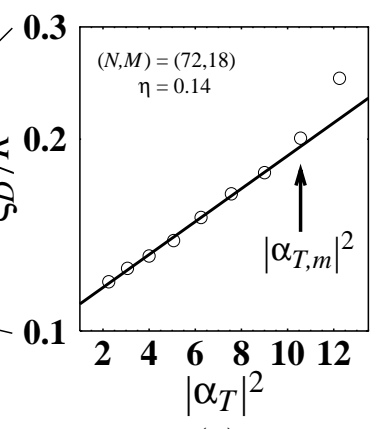

(b)
FIG. 2: In-layer density correlations at layer $M / 2$ of the clean layered-HTSC as the temperature is lowered. (a) Scaled vortex-density correlation-length, $\xi_{D} / R$, for different system sizes at $\eta=0.14$. The dashed curve is just a guide to the eye. (b) The same characteristic length-scale, $\xi_{D} / R$, as a function of $\left|\alpha_{T}\right|^{2}$ in log-linear scale for the system $(N, M)=(72,18)$ with exponential fit (solid line). [The arrow marks the transition temperature for $\eta=0.14, \alpha_{T, m}=-3.25$.]

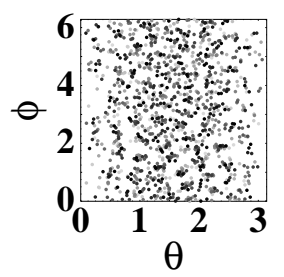

(a)

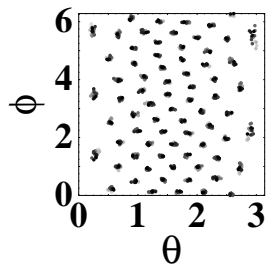

(d)

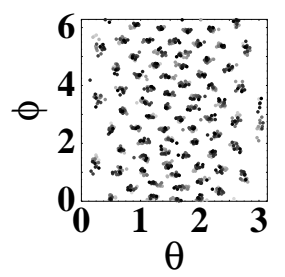

(b)

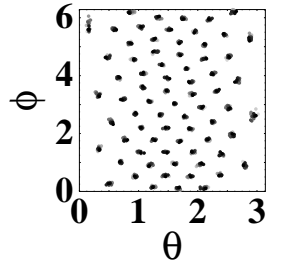

(e)

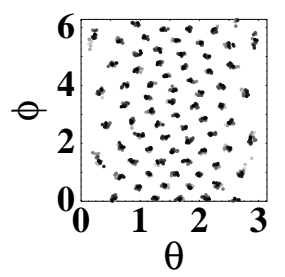

(c)

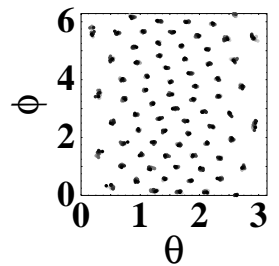

(f)
FIG. 3: Angular vortex-positions in all layers of the pure layered-superconductor with interlayer coupling $\eta=0.14$ for $(N, M)=(72,18)$ as the temperature is lowered: $\alpha_{T}=$ $-3,-4,-5,-6,-7,-8$. [(a),(b),(c),(d),(e),(f) respectively.] Equal gray-tone represent the same layer. The appearance of pancake-vortex domains signals the beginning of the coupled phase [which also appears at a temperature between $\alpha_{T}=-3$ and -4 .

Here the overline denotes a spatial average over the surface of the sphere of radius $R$ and the prefactor is just a "normalization" factor. We impose periodic boundary conditions on the order parameter: $\psi_{M+p}=$ $\psi_{p}, p=1,2, \ldots, M$. Appearance of long-range interlayer correlations can be observed directly from plots of the vortex positions in all layers as the temperature is lowered below the effective temperature $\alpha_{T, m}$ (see Fig. 3) and are confirmed by the change from exponential to algebraic decay of the phase correlation function, $\operatorname{Re}\left[C_{P}(m)\right]$, in Fig. 4(a). In the high-temperature 


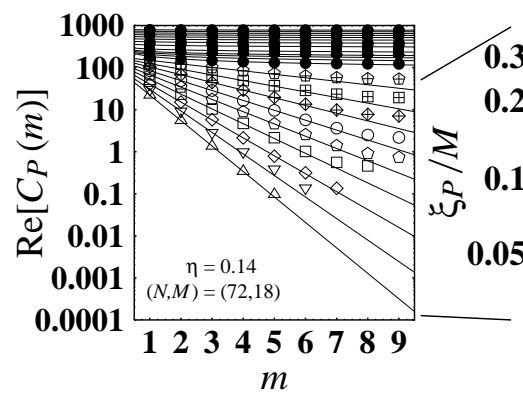

(a)

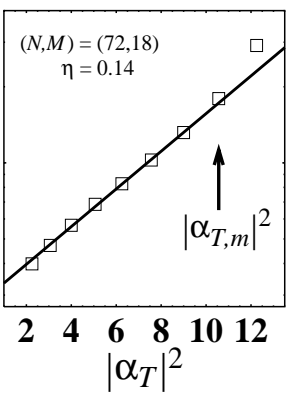

(b)
FIG. 4: Inter-layer phase correlations on the pure layeredHTSC as temperature is lowered. (a) Log-linear plot of the inter-layer phase-correlation-function, $\operatorname{Re}\left[C_{P}(m)\right]$, as a function of the "distance" $m$ along the $c$-axis. Exponential decay is observed above (open symbols) and about (crossed symbols) the transition temperature, $\alpha_{T, m}$, but power-law decrease (filled symbols) below the transition. In each case the mentioned curve is fitted. (b) Scaled phase-correlationlength, $\xi_{P} / M$, vs $\left|\alpha_{T}\right|^{2}$ in log-linear scale for the system in (a) and also with exponential fit. [The arrow marks the coupling transition temperature, which to our resolution coincides with $\left.\alpha_{T, m} \cdot\right]$

regime, where the phase correlation function decays exponentially as $\operatorname{Re}\left[C_{P}(m)\right] \sim \exp \left(-m / \xi_{P}\right)$, we extract the phase correlation-length, $\xi_{P}$, and plot it (scaled by M) against $\left|\alpha_{T}\right|^{2}$ (Fig. 4(b)). We remark that, analogously to $\xi_{D}$, Ref. ${ }^{25}$ also predicts the behavior $\xi_{P} \sim$ $\exp \left(\right.$ const $\left.\left|\alpha_{T}\right|^{2}\right)$. This form gives a consistent fit to the data just above $\alpha_{T, m}$ (solid line in Fig. 4(b)), but for $\alpha_{T} \leq \alpha_{T, m}$ the length scale $\xi_{P}$ grows faster than this exponential. We attribute the even more-rapid increase of $\xi_{P}$ for $\alpha_{T} \leq \alpha_{T, m}$ to an inter-layer coupling transi- tion which takes place also at $\alpha_{T, m}$. This criterion gives us good agreement also with numerical values of Ref. ${ }^{15}$ where the estimated coupling-transition temperature is about $\alpha_{T, m}=-3.5$ for $\eta=0.14$. In that investigation, however, no freezing transition is observed! That is the fundamental difference argued in this paper.

To summarize, we have investigated correlation functions for in-layer structure of the pancake-vortex system and for the coupling of the order parameter in different layers in the S-LD-LLL model. Results from MC simulations, measuring the structure factor in every layer, suggest that there is an effective temperature $\alpha_{T, m}$ below which the pancake-vortex system freezes to a crystalline phase but above which the system behaves as a liquid. This is signaled both by appearance of Bragg peaks in the vortex structure-factor $S(\mathbf{k})$ and the rapid increase of the in-layer vortex-density correlation length, $\xi_{D}$. Simultaneous to this freezing transition there appears a coupling of the order parameter in the different layers which is shown directly from measurements of the vortex positions in every layer and is confirmed by the existence of long-ranged correlations in the order parameter of the distant layers. Explicitly, the exponential decay in correlations along the $c$-axis above the transition temperature $\alpha_{T, m}$ changes to algebraic decrease in the low temperature regime, $\alpha_{T}<\alpha_{T, m}$. The transition is of first order and $\alpha_{T, m}$ depends on the interlayer coupling-parameter $\eta$. The LD-LLL model has shown ${ }^{12,15}$ consistency with the experimental melting-curve in YBCO.

The author would like to thank Prof. Mike Moore for guidance during his $\mathrm{PhD}$ and for critical reading of this manuscript. This work was supported by CONACYT (Mexico) and partially by the University of Manchester.
* Electronic address: zamora@nuclecu. unam.mx

1 J. G. Bednorz and K. A. Müller, Z. Phys. B 64, 189 (1986).

${ }^{2}$ L. V. Shubnikov, V. I. Khotkevich, Y. D. Shepelev, and Y. N. Riabinin, Zh. Eksp. Teor. Fiz. 7, 221 (1937).

3 A. A. Abrikosov, Zh. Eksp. Teor. Fiz. 32, 1442 (1957); Soviet Phys. JETP 5, 1174 (1957).

${ }^{4}$ G. Blatter, M. V. Feigel'man, V. B. Geshkenbein, A. I. Larkin, and V. M. Vinokur, Rev. Mod. Phys. 66, 1125 (1994).

5 I-J. Hwang, R. Šášik, and D. Stroud, Phys. Rev. B 54, 12010 (1996).

${ }^{6}$ H. Pastoriza, M. F. Goffman, A. Arribere, and F. de la Cruz, Phys. Rev. Lett. 72, 2951 (1994).

7 T. Shibauchi, T. Nakano, M. Sato, T. Kisu, N. Kameda, N. Okuda, S. Ooi, and T. Tamegai, Phys. Rev. Lett. 83, 1010 (1999).

8 S. Colson, M. Konczykowski, M. B. Gaifullin, Y. Matsuda, P. Gierlowski, M. Li, P. H. Kes, and C. J. van der Beek, Phys. Rev. Lett. 90, 137002 (2003).

9 X. G. Qiu, V. V. Moshchalkov, and J. Karpinski, Phys. Rev. B 62, 4119 (2000).
10 L. I. Glazman and A. E. Koshelev, Phys. Rev. B 43, 2835 (1991).

11 J. P. Rodriguez, Phys. Rev. B 66, 214506 (2002).

12 J. Hu and A. H. MacDonald, Phys. Rev. B 56, 2788 (1997).

13 G. Mohler and D. Stroud, Phys. Rev. B 62, 14665 (2000).

14 N. K. Wilkin and H. J. Jensen, Europhys. Lett. 40, 423 (1997).

15 A. K. Kienappel and M. A. Moore, Phys. Rev. B 60, 6795 (1999); ibid. 61, 3748 (2000).

16 M. J. W. Dodgson and M. A. Moore, Phys. Rev. B 55, 3816 (1997).

17 W. E. Lawrence and S. Doniach, in Low Temperature Physics, proceedings of the 12th international conference, Kyoto, Japan, edited by E. Kanda, (Keygaku, Tokyo, 1971), p.316.

18 L. D. Landau and V. L. Ginzburg, Zh. Eksp. Teor. Fiz. 20, 1064 (1950) [English translation in Collected Papers of L. D. Landau (Pergamon, Oxford, 1965), p.546].

19 S. M. Roy and V. Singh, Phys. Rev. Lett. 51, 2069 (1983).

20 Here we use the same effective temperature parameter as in Ref. ${ }^{16}$ which is renamed $\alpha_{2 T}$ in Ref. ${ }^{15}$ and is related to 
the $g$ parameter in Ref. ${ }^{12}$ by $\alpha_{T}=\sqrt{2} g$.

21 N. Metropolis, A. W. Rosenbluth, M. N. Rosenbluth, A. H. Teller, and E. Teller, J. Chem. Phys. 21, 1087 (1953).

${ }^{22}$ K. Binder, Rep. Prog. Phys. 60, 487 (1997).

23 P. M. Chaikin and T. C. Lubensky, Principles of Condensed Matter Physics (Cambridge, UK, 1997).

24 We employ the usual definition of one MC step as the up- date of all $v_{n, q}$ coefficients in the system. Actually, because each $v_{n, q}$ is selected randomly to be updated; this implies that a few of them might be updated twice or so, while others might not be chosen in that MC step. This effect, however, is compensated after a large number of MC steps.

25 M. A. Moore, Phys. Rev. B 55, 14136 (1997). 